\title{
在多元醇体系中一锅法合成具有良好储锂性能的介孔碳-锡复合材料
}

\author{
叶亚朱婧怡姚依男王雨果 \\ 吴平唐亚文周益明* 陆天虹
}

(江苏省新型动力电池重点实验室 江苏省生物医药功能材料协同创新中心

南京师范大学化学与材料科学学院 南京 210023)

\begin{abstract}
摘要 以介孔碳( $\mathrm{MC})$ 为导电和支撑介质, 在多元醇体系中通过简便的化学还原方法制备纳米结构的介孔碳一锡 (MC-Sn)复合材料. 采用扫描电子显微镜(SEM)、透射电子显微镜(TEM)和恒电流充放电实验对所得产物的形貌、结构 及电化学性能进行表征. 结果表明, 大量的 $\mathrm{Sn}$ 纳米颗粒均匀且致密地附着在介孔碳上. 作为锂离子电池负极材料, MC-Sn 复合物表现出了较好的循环性能和倍率性能. 例如, 在 $100 \mathrm{~mA} \cdot \mathrm{g}^{-1}$ 的充放电速率下循环 40 圈, 其放电比容量保 持在 $721.5 \mathrm{mAh} \cdot \mathrm{g}^{-1}$; 当充放电速率增大到 $1 \mathrm{~A} \cdot \mathrm{g}^{-1}$ 时，其放电比容量仍高达 $265.8 \mathrm{mAh} \cdot \mathrm{g}^{-1}$. 简单的制备方法和优越的 储锂性能, 使得 MC-Sn 复合材料成为一种理想的高性能锂离子电池负极材料.

关键词 锂离子电池; 负极材料; 介孔碳-锡; 多元醇; 化学还原法
\end{abstract}

\section{One-pot Synthesis of Sn/Mesoporous Carbon Composite in a Polyol System with Well-improved Lithium Storage Capability}

\author{
Ye, Ya Zhu, Jingyi Yao, Yinan Wang, Yuguo \\ Wu, Ping Tang, Yawen Zhou, Yiming* Lu, Tianhong
}

(Jiangsu Key Laboratory of New Power Batteries, Jiangsu Collaborative Innovation Center of Biomedical Functional Materials, School of Chemistry and Materials Science, Nanjing Normal University, Nanjing 210023)

\begin{abstract}
By introducing three-dimensional mesoporous carbon (MC) as a conducting and buffering matrix, MC-Sn composite has been synthesized through a facile chemical reduction approach in a polyol system at the temperature of $170{ }^{\circ} \mathrm{C}$ which is protected by nitrogen atmosphere. Pure Sn nanoparticles were synthesized through the same methodology, but without the addition of MC. The morphology, structure and electrochemical performance of the products have been characterized by scanning electron microscopy (SEM), transmission electron microscopy (TEM), and constant current discharge/charge tests. It is indicated that numerous Sn nanoparticles have been uniformly and densely decorated on the MC matrix. When evaluated as an anode material for lithium-ion batteries (LIBs), the as-prepared MC-Sn composite exhibits markedly improved cycling stability and rate capability compared to pure Sn nanoparticles. For example, a high discharge capacity of $721.5 \mathrm{mAh} \cdot \mathrm{g}^{-1}$ can be retained after 40 discharge/charge cycles at a current density of $100 \mathrm{~mA} \cdot \mathrm{g}^{-1}$ in the potential range of $0.01 \sim 2 \mathrm{~V}$. When tested under the same condition, pure Sn nanoparticles remain only $361.7 \mathrm{mAh}^{\circ} \mathrm{g}^{-1}$. The rate capability of MC-Sn composite was examined in comparison with pure Sn nanoparticles at various current densities from 100 to 200,500 and $1000 \mathrm{~mA} \cdot \mathrm{g}^{-1}$. The MC-Sn composite delivers a discharge capacity of $760.5 \mathrm{mAh} \cdot \mathrm{g}^{-1}$ at a current density of $100 \mathrm{~mA} \cdot \mathrm{g}^{-1}$ after 10 cycles. This value decreases to $555.6 \mathrm{mAh} \cdot \mathrm{g}^{-1}\left(200 \mathrm{~mA} \cdot \mathrm{g}^{-1}\right), 366.2 \mathrm{mAh} \cdot \mathrm{g}^{-1}(500 \mathrm{~mA} \cdot$ $\left.\mathrm{g}^{-1}\right), 265.8 \mathrm{mAh} \cdot \mathrm{g}^{-1}\left(1000 \mathrm{~mA} \cdot \mathrm{g}^{-1}\right)$, and finally returns to $684.3 \mathrm{mAh} \cdot \mathrm{g}^{-1}$ at $100 \mathrm{~mA} \cdot \mathrm{g}^{-1}$. In sharp contrast, the discharge capacities of pure Sn nanoparticles decrease rapidly with the increase of current densities. These results demonstrate that the MC-Sn composite possesses markedly improved rate capability, making it a promising anode for LIBs with high power densities.

Keywords lithium-ion batteries; anode materials; MC-Sn; polyol system; chemical reduction approach
\end{abstract}

1 引言

可充电锂离子电池已经被广泛应用于便携式电子产
品、电动交通工具等领域. 近年来, 为了满足不断拓展的 市场需求, 人们不断致力于寻求具有更加优越性能的负

\footnotetext{
* E-mail: zhouyiming@njnu.edu.cn

Received October 8, 2014; published January 21, 2015.
}

Project Supported by the Industry-Academia Cooperation Innovation Fund Project of Jiangsu Province (No. BY2013001-01), Natural Science Foundation of Jiangsu Province (No. BK20130900), Natural Science Foundation of Jiangsu Higher Education Institutions of China (No. 13KJB150026), Priming Scientific Research Foundation for Advanced Talents in Nanjing Normal University (No. 2013103XGQ0008) and the Program Development of Jiangsu Higher Education Institutions.

项目受江苏省产学研前瞻性研究(No. BY2013001-01)、江苏省自然科学基金(No. BK20130900)、江苏省高校自然科学基金(No. 13KJB150026)、南京 师范大学高层次人才启动基金(No. 2013103XGQ0008)和江苏高校优势学科建设工程项目资助. 
极材料, 来替代传统商业化的较低容量的石墨负极材料 (理论容量为 $372 \mathrm{mAh} \cdot \mathrm{g}^{-1}$ ) $^{[1 \sim 3]}$. 金属锡作为理想的有发 展潜力的负极材料之一, 与石墨相比不仅具有较高的容 量(理论容量为 $990 \mathrm{mAh} \cdot \mathrm{g}^{-1}$ ), 而且因具有比石墨更高的 脱嵌锂电位而具有更高的安全性 ${ }^{[4,5]}$. 但是, 由于锡在脱 嵌锂过程中体积膨胀明显, 从而导致显著的粒子团聚、粉 化, 使得锡在充放电循环过程中容量快速衰减 ${ }^{[6,7]}$.

为了解决容量衰减的问题, 人们提出了许多新颖的 锡基纳米结构化的复合材料. 由于碳基材料具有高的导 电性和良好的体积缓冲效应 ${ }^{[8 \sim 10]}$, 人们通常设计合成纳 米结构的锡和碳复合物来改善锡的电化学性能. 已见报 道的与金属锡复合的碳基材料有无定型碳 ${ }^{[11 ~ 15], ~}$ 碳纳 米管 ${ }^{[3,16]}$ 和石墨烯 ${ }^{[6 ~ 18]}$. 相比于纯锡, 这些复合材料在 电化学性能方面均有显著提高.

介孔碳( $\mathrm{MC}$ )作为一种三维多孔碳载体材料, 有更 高的机械强度和更好的电子传输能力 ${ }^{[19-22]}$, 因此, 金属 锡与三维介孔碳的复合材料有望表现出优越的循环性 能和倍率性能. 本文报道了一种在多元醇体系中通过原 位化学还原锡盐制备锡和介孔碳的复合材料的方法, 研 究了介孔碳对金属锡的电化学性能的影响. 实验结果显 示, 介孔碳的引入可以促使形成更小尺寸的锡纳米颗 粒, 与纯锡相比, 介孔碳与锡的复合材料(MC-Sn)表现 出更好的电化学性能, 显示出该材料是一种理想的锂离 子电池负极材料.

\section{2 结果与讨论}

\section{$2.1 \mathrm{MC}-\mathrm{Sn}$ 复合物的形貌和结构特征}

图 1 是 MC-Sn 复合物(曲线 $1 \mathrm{a}$ )和纯 $\mathrm{Sn}$ (曲线 $1 \mathrm{~b}$ )的 XRD 图. 由曲线 $1 b$ 可见, 所制得的纯 $\mathrm{Sn}$ 与 $\mathrm{Sn}$ 的标准 卡片(JCPDS No. 04-0673)完全吻合, 且结晶性良好. 而 在 MC-Sn 复合物中(曲线 $1 \mathrm{a}$ ), 除在 $26^{\circ}$ 处出现 $\mathrm{MC}$ 的特 征衍射峰[对应于 $\mathrm{C}$ 的(002)晶面]外, 无其它衍射峰, 表 明复合物中的 $\mathrm{Sn}$ 为无定型.

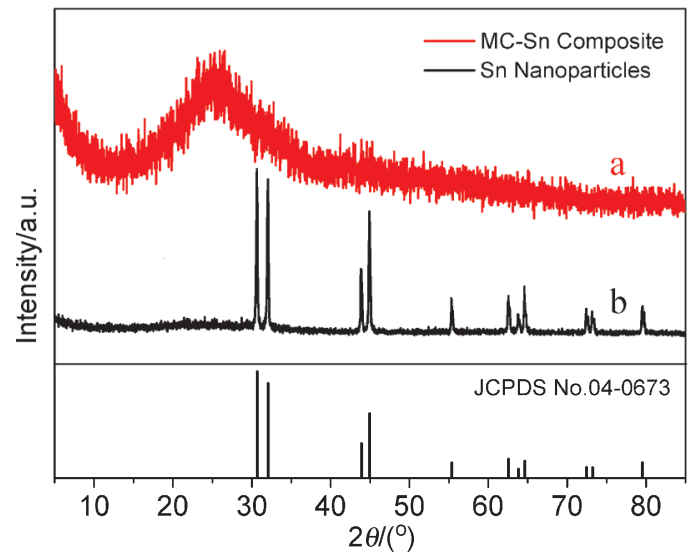

图 $1 \mathrm{MC}-\mathrm{Sn}$ 复合物(曲线 a) 和锡纳米颗粒(曲线 b) 的 XRD 图 Figure 1 XRD patterns of MC-Sn composite (curve a) and Sn nanoparticles (curve b)
图 2 是 MC-Sn 复合物的 SEM 图(2a,b)和 TEM 图(2c, d). 从图 $2 \mathrm{a}, \mathrm{b}$ 中可以看到, 有许多纳米粒子附着在相互 连接的三维多孔结构的 $\mathrm{MC}$ 上. 这可能是由于在实验过 程的加热步骤中, $\mathrm{Sn}^{2+}$ 被吸附到含有丰富羧酸基团的 $\mathrm{MC}$ 表面, 滴加 $\mathrm{NaBH}_{4}$ 后由于静电作用, 吸引带负电的 $\mathrm{BH}_{4}$ 离子, 这样 $\mathrm{Sn}^{2+}$ 慢慢的被还原成 $\mathrm{Sn}$ 核, 最后形成 $\mathrm{Sn}$ 粒子均匀的分布在 $\mathrm{MC}$ 上. 图 2c, d 显示了 $\mathrm{MC}$ 的多 孔形貌, 许多 $\mathrm{Sn}$ 纳米粒子点缀在 $\mathrm{MC}$ 上, 在 $\mathrm{MC}$ 以外的 区域无多余的 $\mathrm{Sn}$ 纳米粒子.

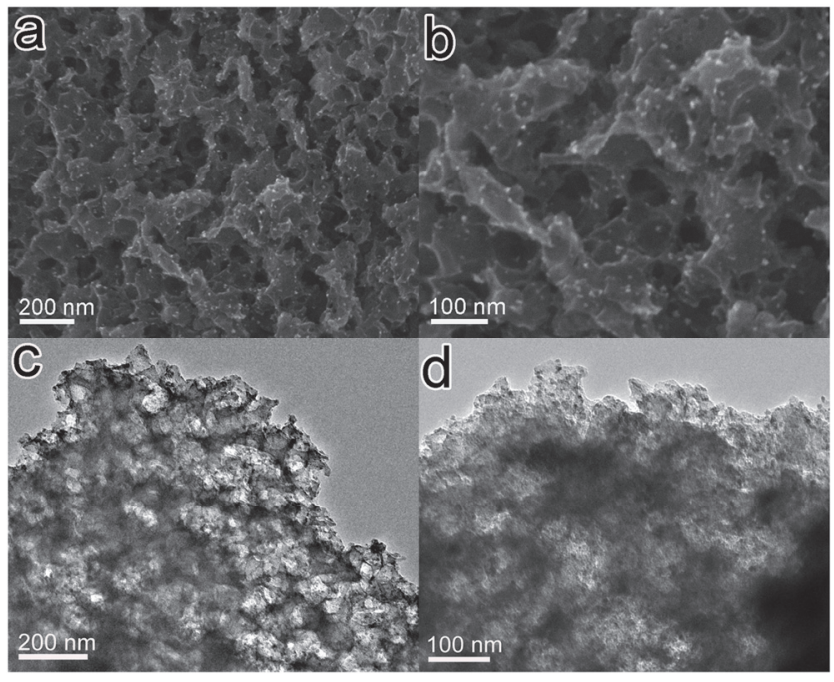

图 $2 \mathrm{MC}-\mathrm{Sn}$ 复合物在不同放大倍数下的 $\operatorname{SEM}$ 图 $(\mathrm{a}, \mathrm{b})$ 和 TEM 图 $(\mathrm{c}, \mathrm{d})$ Figure 2 (a, b) SEM and (c, d) TEM images of the MC-Sn composite with different magnifications

图 $3 b$ 清楚地给出了复合物中 $\mathrm{C}$ 元素(黄色)和 $\mathrm{Sn}$ 元 素(绿色)的分布情况, 观察到的元素分布信号只出现在 选定的区域内，即对应于图 3a 的 TEM 图区域. 可见， $\mathrm{MC}-\mathrm{Sn}$ 中的 $\mathrm{Sn}$ 纳米颗粒是均匀分布于 $\mathrm{MC}$ 中的.

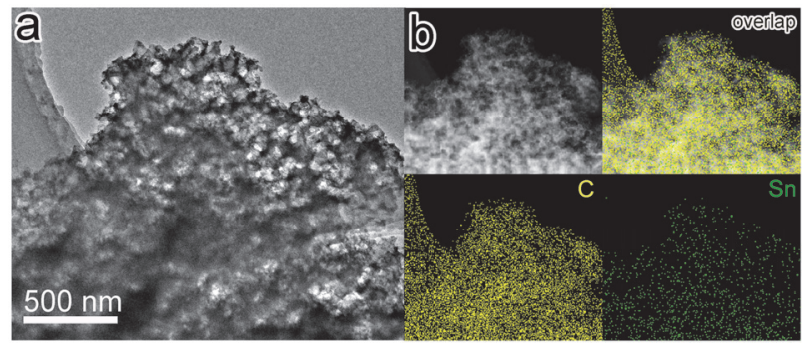

图 3 (a) MC-Sn 复合物的 TEM 图; (b)相应区域的元素分布图, 黄色 代表 C 元素, 绿色代表 $S n$ 元素

Figure 3 (a) TEM image and (b) its corresponding elemental mappings of C (yellow), Sn (green), and their overlap of the MC-Sn composite

为了作对比, 纯 $\mathrm{Sn}$ 纳米颗粒的制备与 $\mathrm{MC}-\mathrm{Sn}$ 复合 物制备实验过程相同，只是未加入 MC. 图 4 是纯 $\mathrm{Sn}$ 纳 米颗粒在不同放大倍数下的 SEM 图, 可见纯 Sn 颗粒尺 寸不很均匀, 且较图 $2 \mathrm{a}, \mathrm{b}$ 显示的 $\mathrm{Sn}$ 粒子的尺寸大得多. 这些信息表明, $\mathrm{MC}$ 的引入有利于形成尺寸更小、粒子均 
匀的 $\mathrm{Sn}$ 纳米颗粒.

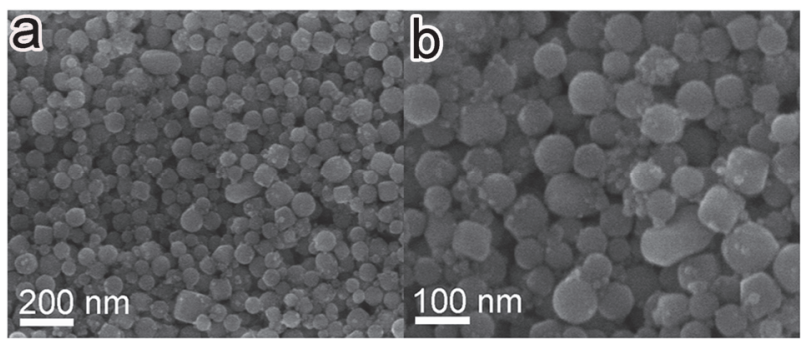

图 $4 \mathrm{Sn}$ 纳米颗粒在不同放大倍数下的 SEM 图

Figure 4 SEM images of the Sn nanoparticles with different magnifications

碳基复合物中, 碳的含量与材料的储锂性能相 关 ${ }^{[5,7,11]}$. 为了确定 $\mathrm{MC}-\mathrm{Sn}$ 复合物中碳的含量, 我们对 $\mathrm{MC}-\mathrm{Sn}$ 复合物进行了热重分析. 图 5 是 MC-Sn 复合物 在空气氛中的热重 $(\mathrm{TG})$ 曲线. 从图中可以看到, 在 $200 \sim 500{ }^{\circ} \mathrm{C}$ 温度范围内复合物的失重 $42.2 \mathrm{wt} \%$, 该失 重来自于 $\mathrm{MC}$ 的氧化燃烧. 因此, 该复合物中 $\mathrm{MC}$ 的含 量为 $42.2 \mathrm{wt} \%$.

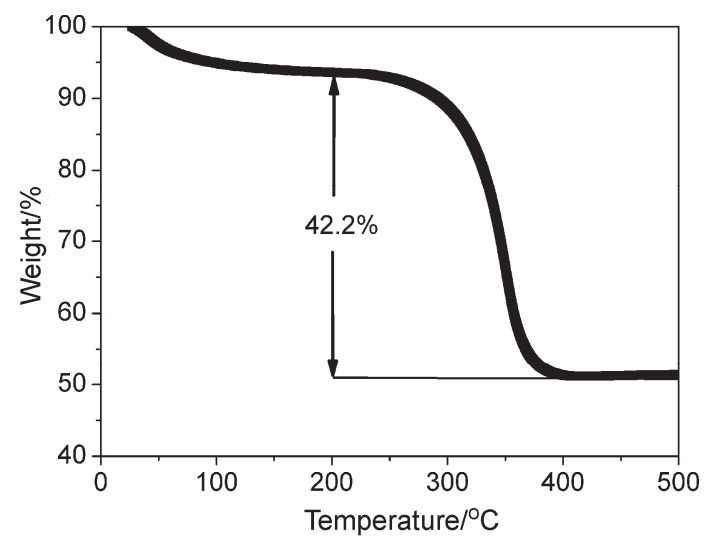

图 $5 \mathrm{MC}-\mathrm{Sn}$ 复合物的热重曲线

Figure 5 TG curve of the MC-Sn composite

\section{$2.2 \mathrm{MC}-\mathrm{Sn}$ 复合物的电化学性能}

图 6 是在 $0.01 \sim 2 \mathrm{~V}$ 的电位区间、 $100 \mathrm{~mA} \cdot \mathrm{g}^{-1}$ 的充 放电电流密度下, MC-Sn 复合物与纯 $\mathrm{Sn}$ 纳米颗粒的放 电比容量随着循环次数的变化图. 当循环 40 圈以后, $\mathrm{MC}-\mathrm{Sn}$ 复合物的放电容量为 $721.5 \mathrm{mAh} \cdot \mathrm{g}^{-1}$, 而纯 $\mathrm{Sn}$ 放 电容量仅为 $361.7 \mathrm{mAh} \cdot \mathrm{g}^{-1}$. 与纯 $\mathrm{Sn}$ 相比, $\mathrm{MC}-\mathrm{Sn}$ 复合 物显示出了较好的循环性能和较高的比容量. 这可能是 由于具有高机械强度的三维多孔 $\mathrm{MC}$ 的引入, 不仅提高 了材料的电子导电能力, 还促进形成尺寸更小的 Sn 颗 粒均匀分散在介孔碳上. 在充放电过程中, 颗粒有一定 的体积膨胀缓和空间, 有效地抑制了材料的团聚和粉 化, 从而大大提高了材料的充放电循环性能, 改善了电 池容量的衰减问题.

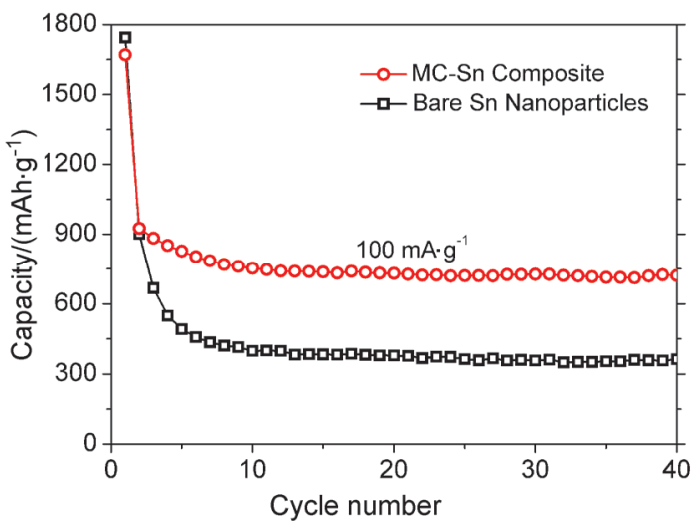

图 $6 \mathrm{Sn}$ 纳米粒子和 MC-Sn 复合物的放电比容量随着循环次数的变 化图, 充放电电流密度为 $100 \mathrm{~mA} \cdot \mathrm{g}^{-1}$, 充放电区间为 $0.01 \sim 2 \mathrm{~V}$

Figure 6 Cycling performance of the Sn nanoparticles and MC-Sn composite at a current density of $100 \mathrm{~mA} \cdot \mathrm{g}^{-1}$ in the potential range of $0.01 \sim 2 \mathrm{~V}$

图 7 是 $\mathrm{MC}-\mathrm{Sn}$ 复合物的前五圈循环伏安曲线. 在首 次循环过程中, $0.5 \mathrm{~V}$ 的还原峰可归结于嵌锂过程中固 体电解质界面(SEI)膜的形成. Sn 负极材料的脱嵌锂机 制不同于石墨材料，它是通过可逆地与锂形成 $\mathrm{Li}_{x} \mathrm{Sn}$ 合 金的方式来储锂的，其储锂机理可表示为 ${ }^{[4]}$ :

$$
\mathrm{Sn}+x \mathrm{Li}^{+}+x \mathrm{e}^{-} \leftrightarrow \mathrm{Li}_{x} \mathrm{Sn} \quad(0 \leqslant x \leqslant 4.4)
$$

图 7 中 $0.0 \sim 0.4 \mathrm{~V}$ 的还原峰对应的是 $\mathrm{Sn}$ 与 $\mathrm{Li}$ 形成 $\mathrm{Li}_{x} \mathrm{Sn}$ 合金的过程.

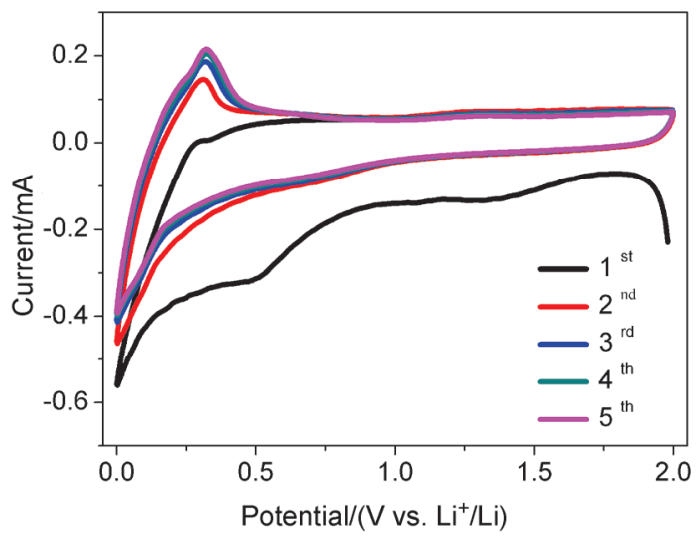

图 $7 \mathrm{MC}-\mathrm{Sn}$ 复合物前五圈的循环伏安曲线，电位区间为 $0.0 \sim 2 \mathrm{~V}$, 电位扫速为 $0.2 \mathrm{mV} \cdot \mathrm{s}^{-1}$

Figure 7 The first five CV curves of the MC-Sn composite at a scan rate of $0.2 \mathrm{mV} \cdot \mathrm{s}^{-1}$ in the potential range of $0.0 \sim 2 \mathrm{~V}$

图 8 是 MC-Sn 复合物在第 1、2、10、20、40 圈的 充放电曲线图. 从图中可以看出, $\mathrm{MC}-\mathrm{Sn}$ 复合物的脱嵌 锂平台与 $\mathrm{CV}$ 中的氧化还原峰位相对应, 但较已报道的 文献偏低 ${ }^{[13,15]}$, 这可能是由于 $\mathrm{Sn}$ 与 $\mathrm{MC}$ 的协同作用的结 果. 第 10、20、40 圈的充放电容量几乎保持不变, 这说 明了 MC-Sn 复合物具有良好的循环稳定性. 


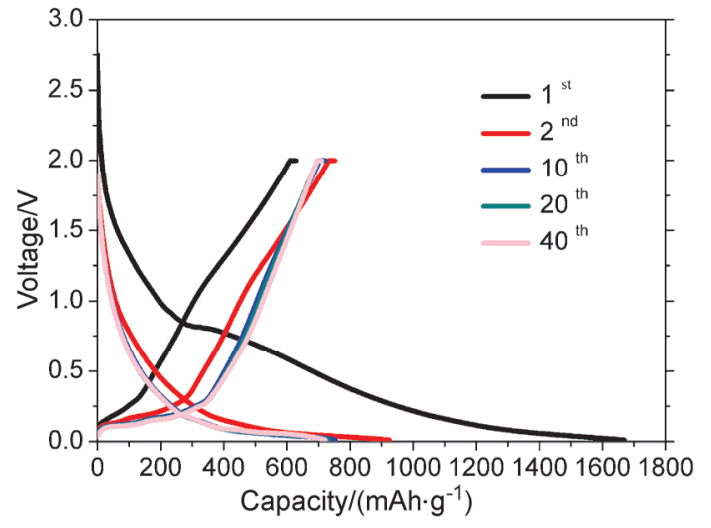

图 $8 \mathrm{MC}-\mathrm{Sn}$ 复合物在第 $1 、 2 、 10 、 20 、 40$ 圈的充放电曲线图, 充放 电电流密度为 $100 \mathrm{~mA} \cdot \mathrm{g}^{-1}$, 充放电区间为 $0.01 \sim 2 \mathrm{~V}$

Figure 8 The discharge and charge profiles of the MC-Sn composite for the 1st, 2nd, 10th, 20th and 40th cycle at a current density of $100 \mathrm{~mA} \cdot \mathrm{g}^{-1}$ in the potential range of $0.01 \sim 2 \mathrm{~V}$

图 9 是 $\mathrm{Sn}$ 纳米粒子和 $\mathrm{MC}-\mathrm{Sn}$ 复合物在 $0.01 \sim 2 \mathrm{~V}$ 电位区间, 不同充放电电流密度下, 放电比容量随着循 环次数的变化图 $(1 \sim 50$ 圈). 当充放电电流密度从 100 $\mathrm{mA} \cdot \mathrm{g}^{-1}$ 增加到 200、500 和 $1000 \mathrm{~mA} \cdot \mathrm{g}^{-1}$ 时, $\mathrm{MC}-\mathrm{Sn}$ 复 合物的平均放电比容量从 $760.5 \mathrm{mAh} \cdot \mathrm{g}^{-1}$, 下降到 555.6、366.2、265.8 $\mathrm{mAh} \cdot \mathrm{g}^{-1}$; 当充放电电流密度再回 到 $100 \mathrm{~mA} \cdot \mathrm{g}^{-1}$ 时, 放电比容量仍然能够回到 684.3 $\mathrm{mAh} \cdot \mathrm{g}^{-1}$. 而 $\mathrm{Sn}$ 纳米粒子在不同倍率下的放电比容量均 显著低于 MC-Sn 复合物, 甚至在放电电流密度为 1000 $\mathrm{mA} \cdot \mathrm{g}^{-1}$ 时放电容量几乎为零. 这一结果说明, 在较高的 充放电倍率下 MC-Sn 复合物较 $\mathrm{Sn}$ 纳米粒子具有更好的 耐大电流充放电性能, 预示着 MC-Sn 复合物非常有希 望成为高功率密度锂离子电池的负极材料.

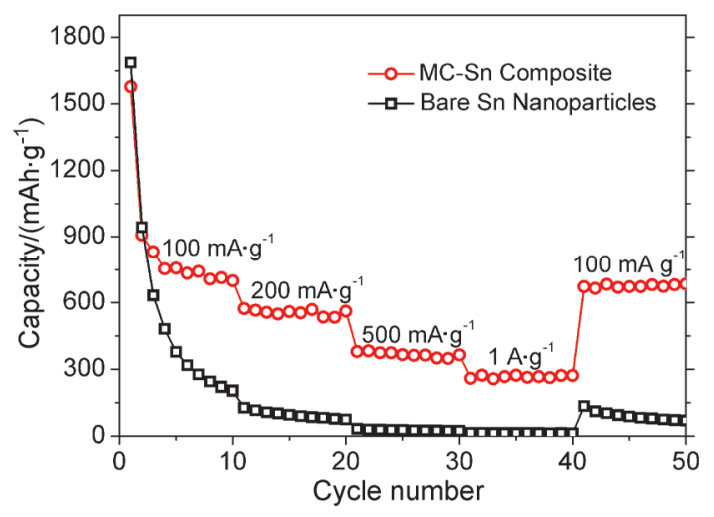

图 9 在 $0.01 \sim 2 \mathrm{~V}$ 的电位区间内 $\mathrm{Sn}$ 纳米粒子和 MC-Sn 复合物的放 电倍率性能图

Figure 9 Rate capability of the Sn nanoparticles and MC-Sn composite at different current densities in the potential range of $0.01 \sim 2 \mathrm{~V}$

\section{3 结论}

以 $\mathrm{MC}$ 为碳基介质, 在多元醇体系中通过原位化学 还原的方法制备 MC-Sn 复合材料. 由于 $\mathrm{MC}$ 的引入可以
提高材料的稳定性和电子导电性, 因此, 与纯 $\mathrm{Sn}$ 相比, MC-Sn 复合材料表现出了较好的循环性能和倍率性能: 在 $100 \mathrm{~mA} \cdot \mathrm{g}^{-1}$ 的充放电电流密度下, 40 次循环后 MC-Sn 复合物的放电比容量保持在 $721.5 \mathrm{mAh} \cdot \mathrm{g}^{-1}$; 而 在 $1 \mathrm{~A} \cdot \mathrm{g}^{-1}$ 的高充放电电流密度下, 放电比容量仍高达 $265.8 \mathrm{mAh} \cdot \mathrm{g}^{-1}$. 因此, MC-Sn 复合物是一种理想的锂离 子电池负极材料.

\section{4 实验部分}

\subsection{MC-Sn 复合物的制备}

$\mathrm{MC}$ 的酸化处理: $\mathrm{MC}$ 是以纳米 $\mathrm{CaCO}_{3}$ 为模板, 葡萄 糖为碳源通过高温热解及随后的去除模板过程制备得 到 ${ }^{[18,19]}$. 将制得的 $\mathrm{MC}$ 置于浓硝酸 $(65 \mathrm{wt} \%)$ 中, 油浴中 加热回流 $5 \mathrm{~h}$ 后, 用蒸馏洗涤至中性, $60{ }^{\circ} \mathrm{C}$ 空气氛中干 燥得到酸处理的 MC.

MC-Sn 复合物的制备: 将 $20 \mathrm{mg}$ 酸化处理的 MC 和 $5 \mathrm{~g}$ 聚乙烯基吡咯烷酮(PVP, $\mathrm{k} 30$ )分散于 $100 \mathrm{~mL}$ 的一缩 二乙二醇( $\mathrm{DEG}, \mathrm{C}_{4} \mathrm{H}_{10} \mathrm{O}_{3}$ ) 中, 再向溶液中加入 $0.3 \mathrm{mmol}$ $\mathrm{SnCl}_{2} \cdot 2 \mathrm{H}_{2} \mathrm{O}$. 油浴中加热, 待溶液温度升至 $170{ }^{\circ} \mathrm{C}$, 向 体系中滴加 $10 \mathrm{~mL} 1 \mathrm{~mol} \cdot \mathrm{L}^{-1} \mathrm{NaBH}_{4}$ 的 DEG 溶液. 在 $\mathrm{N}_{2}$ 气氛中 $170{ }^{\circ} \mathrm{C}$ 恒温 $1 \mathrm{~h}$ 以后用水和乙醇离心洗涤, $80{ }^{\circ} \mathrm{C}$ 条件下真空干燥. 最后, 在管式炉中 $\mathrm{N}_{2}$ 气氛下以 $1{ }^{\circ} \mathrm{C} \cdot \min ^{-1}$ 的升温速度升温至 $400{ }^{\circ} \mathrm{C}$, 并在此温度下裆 烧 $2 \mathrm{~h}$. 之后, 随炉自然冷却至室温便得 MC-Sn 复合物.

$\mathrm{Sn}$ 纳米粒子的制备: 除不加 $\mathrm{MC}$ 外，其余均同于上 述复合物的制备.

\section{2 电极的制备及电池的组装}

电极的制备: 将 MC-Sn 复合物、乙炔黑、聚偏氟乙 烯(PVDF)按 $80: 10: 10$ 的质量比混合, 混合物在玛瑙 研针中研磨均匀后, 分散在 $N$-甲基吡咯烷酮(NMP)中, 搅拌均匀后涂于泡沫铜集流体上, 在 $120{ }^{\circ} \mathrm{C}$ 下真空干燥 $12 \mathrm{~h}$.

电池的组装: 在氩气保护的手套箱(IL-2GB, Innovative Technology) 中, 将上述涂有活性物质的集流体组 装成纽扣电池(CR2025), 金属锂片为对电极, 聚丙烯多 孔薄膜 (Celgard 2400 隔膜)为隔膜, 电解质溶液为 1 $\mathrm{mol} \cdot \mathrm{L}^{-1}$ 的六氟磷酸锂 $\left(\mathrm{LiPF}_{6}\right)$ 的碳酸乙烯酯 $(\mathrm{EC})$ 和碳酸 二甲酯(DMC)(体积比 $1: 1$ ) 溶液. 所有电化学测量均在 $20{ }^{\circ} \mathrm{C}$ 下进行, 计算比容量时是将 MC-Sn 作为整体来考 虑的.

\section{References}

[1] Xu, Y.; Guo, J.; Wang, C. J. Mater. Chem. 2012, $22,9562$.

[2] Wang, C.; Li, Y.; Chui, Y. S.; Wu, Q. H.; Chen, X.; Zhang, W. Nanoscale 2013, $5,10599$.

[3] Uysal, M.; Cetinkaya, T.; Alp, A.; Akbulut, H. Appl. Surf. Sci. 2014, $290,6$.

[4] Derrien, G.; Hassoun, J.; Panero, S.; Scrosait, B. Adv. Mater. 2007, 19,2336

[5] Zhang, W. M.; Hu, J. S.; Guo, Y. G.; Zheng, S. F.; Zhong, L. S.; 
Song, W. G.; Wan, L. Adv. Mater. 2008, 20, 1160.

[6] Li, N.; Song, H.; Cui, H.; Wang, C. Nano Energy 2014, 3, 102.

[7] Wu, P.; Du, N.; Liu, J.; Zhang, H.; Yu, J.; Yang, D. R. Mater. Res. Bull. 2011, 46, 2278.

[8] Liu, X.; Xie, J. Y.; Zhao, H. L.; Wang, K.; Tang, W. P.; Pan, Y. L.; Feng, Z. H.; Lv, P. P. Acta Chim. Sinica 2013, 71, 1011. (刘欣, 解晶 莹, 赵海雷, 王可, 汤卫平, 潘延林, 丰震河, 吕鹏鹏, 化学学报, 2013, 71, 1011.)

[9] Feng, R.; Wang, L. W.; Lv, Z. Y.; Wu, Q.; Yang, L. J.; Wang, X. Z.; $\mathrm{Hu}$, Z. Acta Chim. Sinica 2014, 72, 653. (冯瑞, 王立伟, 吕之阳, 吴强, 杨立军, 王喜章, 胡征, 化学学报, 2014, 72, 653.)

[10] Dai, L. Q.; Wu, F.; Guan, Y. B.; Fu, K.; Jin, Y.; Gao, W.; Wang, Z.; Su, Y. F. Acta Chim. Sinica 2014, 72, 583. (戴丽琴, 吴锋, 官亦标, 傅凯，金翼，高伟，王昭，苏岳锋，化学学报, 2014, 72, 583.)

[11] Chen, J.; Yang, L.; Fang, S.; Zhang, Z.; Deb, A.; Hirano, S. I. Electrochim. Acta 2014, 127, 390 .

[12] Hassoun, J.; Derrien, G.; Panero, S.; Scrosait, B. Adv. Mater. 2008, $20,3169$.

[13] Yu, Y.; Gu, L.; Wang, C.; Dhanabalan, A.; Aken, P. A.; Maier, J. Angew. Chem. Int. Ed. 2009, 48, 6485.
[14] Wang, J.; Li, D.; Fan, X.; Gou, L.; Wang, J.; Li, Y.; Lu, X.; Li, Q. J. Alloys Compd. 2012, 516, 33.

[15] Zhu, Z.; Wang, S.; Du, J.; Jin, Q.; Zhang, T.; Chen, J. Nano Lett. 2014, 14, 153.

[16] Seo, S. D.; Lee, G. H.; Lim, A. H.; Min, K. M.; Kim, J. C.; Shim, H. Y.; Park, K. S.; Kim, D. W. RSC Adv. 2012, 2, 3315.

[17] Wang, G.; Wang, B.; Wang, X.; Park, J.; Dou, S.; Ahn, H.; Kim, K. J. Mater. Chem. 2009, 19, 8378.

[18] Wang, D.; Li, X.; Yang, J.; Wang, J.; Geng, D.; Li, R.; Cai, M.; Sham, T. K.; Sun, X. Phys. Chem. Chem. Phys. 2013, 15, 3535

[19] Li, J.; Wu, P.; Lou, F.; Zhang, P.; Tang, Y.; Zhou, Y.; Lu, T. Electrochim. Acta 2013, 111, 862 .

[20] Shen, L.; Zhang, X.; Uchaker, E.; Yuan, C.; Cao, G. Adv. Energy Mater. 2012, 2, 691.

[21] Chen, L.; Wu, P.; Xie, K.; Li, J.; Xu, B.; Cao, G.; Chen, Y.; Tang, Y.; Zhou, Y.; Lu, T.; Yang, Y. Electrochim. Acta 2013, 92, 433.

[22] Xu, B.; Peng, L.; Wang, G.; Cao, G.; Wu, F. Carbon 2010, 48, 2377.

[23] Xu, B.; Shi, L.; Guo, X.; Peng, L.; Wang, Z.; Chen, S.; Cao, G.; Wu, F.; Yang, Y. Electrochim. Acta 2011, 56, 6464. 